\title{
Measurement of Light Intensity Based on a Flip-flop Sensor
}

M. Kollár

This paper deals with a new type of system for measuring light intensity with the use of a flip-flop sensor controlled by a so-called slow-rise voltage control pulse. A photodiode was used for quantification of the measured light intensity in the structure of the flip-flop. The theoretical considerations are compared with experimental results, and good agreement is reported.

Keywords: measurement, flip-flop sensor, light intensity, equivalent voltage.

\section{Introduction}

The circuit in Fig. 1 was introduced in [1] as a flip-flop sensor. The flip-flop sensor is part of a class of silicon sensors with a digital output. A standard flip-flop consisting from two transistors and two resistors (see Fig. 1) is characterized by two stable states, 1 and 0 .

One of the authors of the patent flip-flop sensor was Lian [1], who showed that a flip-flop sensor can be used for measuring non-electrical quantities and derived a formula for calculating the equivalent voltage of the flip-flop sensor controlled by a slow-rise control pulse. The principle of measurement is based on the measured non-electrical quantity breaking the value symmetry of the inverters relative to the morphological symmetry axis passing through points $\mathrm{K}$ and Z (see Fig. 1).

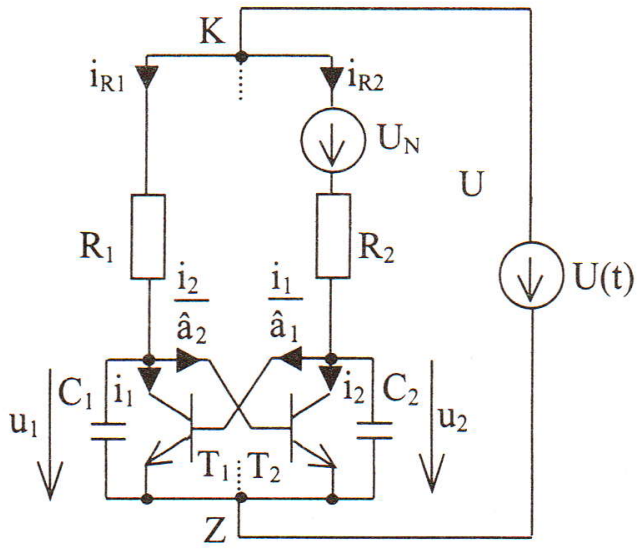

Fig. 1: Flip-flop sensor

However measured quantity can be compensated by a voltage $U_{N}=U_{N E}$ in such a way that by repeated connection to source $U(t)$ the $50 \%$ state [1] is restored, so that the magnitude of the measured non-electrical quantity will be reflected in the voltage $U_{N E}$, which we will call the equivalent voltage. However, it is not necessary to stick to the custom of using sensorial elements, as shown in Fig. 1.

It should be noted that in voltage control we also distinguish between pulses with a fast or slow-rise segment of the control pulse (see Fig. 2).

Control with a slow-rise segment of the control pulse is characterized by the ratio $U_{m} / \delta_{I}$ being such that the currents

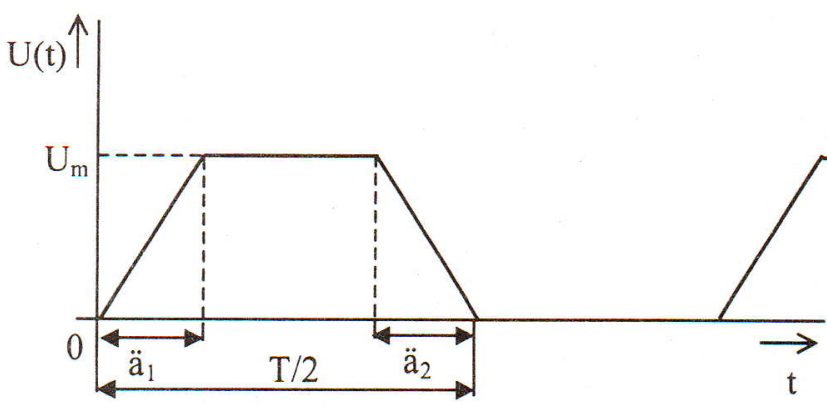

Fig. 2: Voltage control pulse

passing through the capacitors are negligible compared to the transistor currents of the flip-flop sensors. The notion negligible should be understood in its relative sense. In practice, this condition is satisfied if $\delta_{1}, \delta_{2} \gg R_{1} C_{1}$ and $\delta_{1}, \delta_{2} \gg R_{2} C_{2}$ at the same time. The goal of this paper is to show that light intensity can be measured with the use of a flip-flop sensor.

\section{Equivalent voltage}

As described above, the asymmetry of the flip-flop sensor can be compensated by the equivalent voltage $U_{N E}$ [1]. If we assume mismatches in the load resistors $R_{1}, R_{2}$, saturation currents $i_{E S 1}, i_{E S 2}$ or mismatches in the current coefficient $\beta_{1}$, $\beta_{2}$ of the transistors of the flip-flop sensor, then the formula for the equivalent voltage has the form $[1,2]$ :

$$
U_{N E}=V_{T} \ln \left[\frac{\left(R_{1}-\frac{R_{2}}{\beta_{1}}\right)}{\left(R_{2}-\frac{R_{1}}{\beta_{2}}\right)} \frac{i_{E S 1}}{i_{E S 2}}\right]
$$

A flip-flop circuit with a photodiode for measuring light intensity is shown in Fig. 3.

In this case, the value of the equivalent voltage can be calculated using formula (2):

$$
U_{N E}=I R+V_{T} \ln \left[\frac{\left(R_{1}^{\prime}-\frac{R_{2}}{\beta_{1}}\right)}{\left(R_{2}-\frac{R_{1}^{\prime}}{\beta_{2}}\right)} \frac{i_{E S 1}}{i_{E S 2}}\right]
$$

where $R_{1}^{\prime}=R_{1}+R, V_{T}$ is a thermal voltage and $R \ll R_{1}$. In equation (2), I represents the photo-current caused by a mea- 


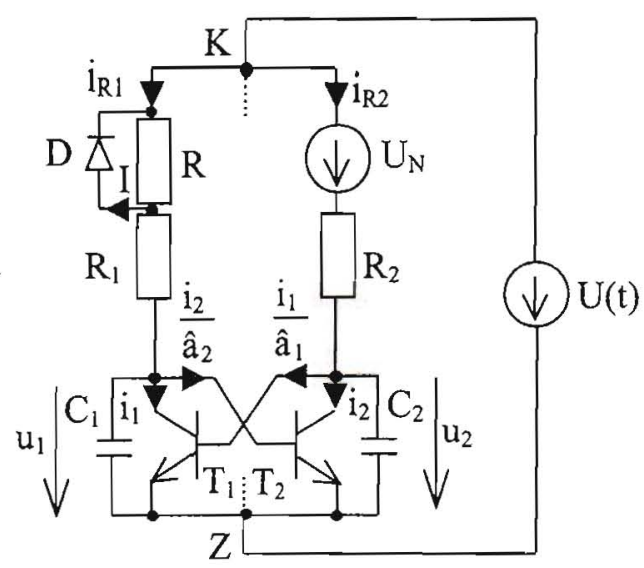

Fig. 3: Flip-flop sensor with a photodiode $D$

sured light intensity. Let us assume that the bipolar transistors of the flip-flop were matched, as were the load resistors. Then the part of the equivalent voltage caused by any disturbances and calculated by equation ( 1 ) is negligible compared to the expression $I R$ in (2). However, this must be understood in its relative sense, because the assumption is not valid if the value of the equivalent voltage into which the disturbances and the value corresponding to the light intensity are reflected are from same range. As shown in section 3 "Proposed solution and experimental results", the contribution of this inaccuracy to the relative error in the result as a function of the light intensity may be particularly relevant in the lower part of the range.

\section{Proposed solution and experimental results}

Fig. 4 shows a system for measuring light intensity. Here the feedback is fed from the outputs of the flip-flop to the collector resistor $R_{2}$. As can be seen, in Fig. 4, sampling circuit $\mathrm{S} / \mathrm{H}$, the reverse counter and DAC are connected in feedback. It is necessary to distinguish between the controlling pulses, with the symbol $\Phi$ in Fig. 4, and the controlling pulses with the shape shown in Fig. 2. The pulses $\Phi$ control the digital circuits as sampling circuits, reverse counter and DAC, while the pulses with the shape in Fig. 2 are needed for correct functioning of the flip-flop.

The principle of operation is based on the measured light intensity breaking the value symmetry of the flip-flop, but as shown above this can be compensated by the equivalent voltage. In this case, the asymmetry will be reflected in the number of pulses read by the reverse counter. The light intensity as a function of time will be equivalent to the number of the pulses read as function of time.

As known, the dependence between the voltage, given by expression $I R$, and the light intensity is in general non-linear. This also predicts a non-linear dependence between the light intensity and number of pulses read. One way is to use a polynomial function, the coefficient of which can be found using the well-known least squares method. In our experiment, a polynomial function was used, as follows

$$
\begin{aligned}
E & =a_{0}+a_{1} U_{N E}+a_{2} U_{N E}^{2}+a_{3} U_{N E}^{3}+ \\
& +a_{4} U_{N E}^{4}+a_{5} U_{N E}^{5}+a_{6} U_{N E}^{6},
\end{aligned}
$$

where $E$ represents light intensity, $U_{N E}=I R$ and coefficients: $a_{1}=0.1100 \mathrm{~lx} / \mathrm{V}, a_{2}=5.1936 \mathrm{~lx} / \mathrm{V}^{2}, a_{3}=-0.6859 \mathrm{~lx} / \mathrm{V}^{3}$, $a_{4}=0.0467 \mathrm{~lx} / \mathrm{V}^{4}, a_{5}=-0.0012 \mathrm{~lx} / \mathrm{V}^{5}, a_{6}=1.0082 \cdot 10^{-5} \mathrm{~lx} / \mathrm{V}^{6}$.

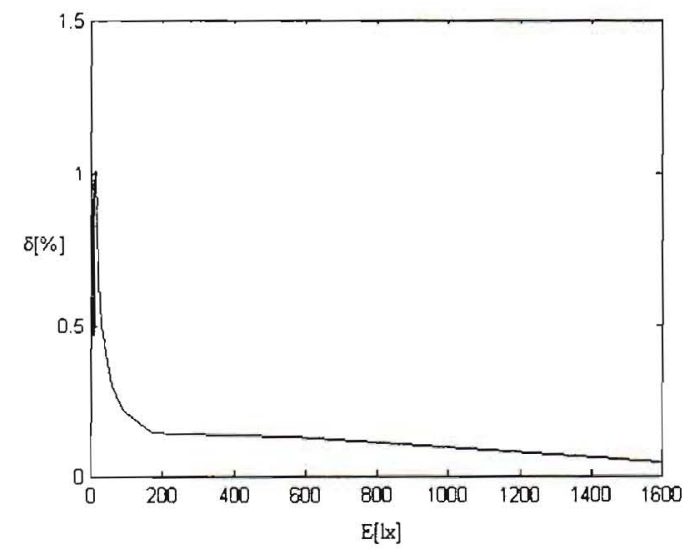

Fig. 5: Relative error as a function of $E$

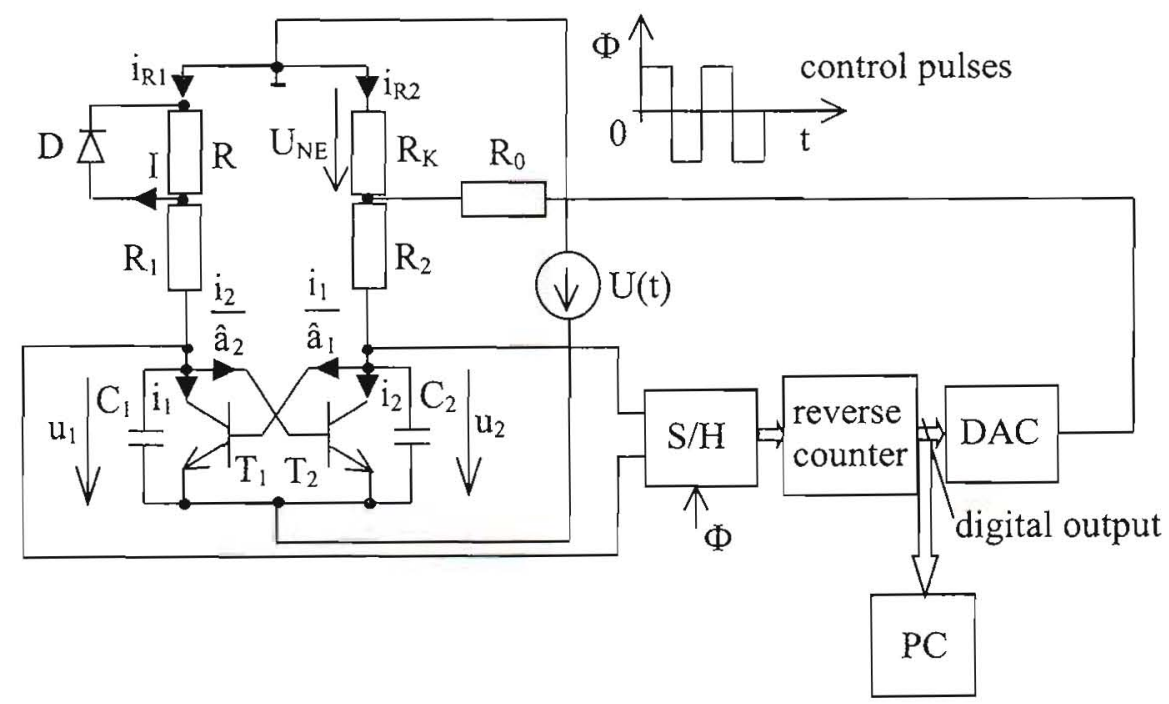

Fig. 4: The complete sensor system 


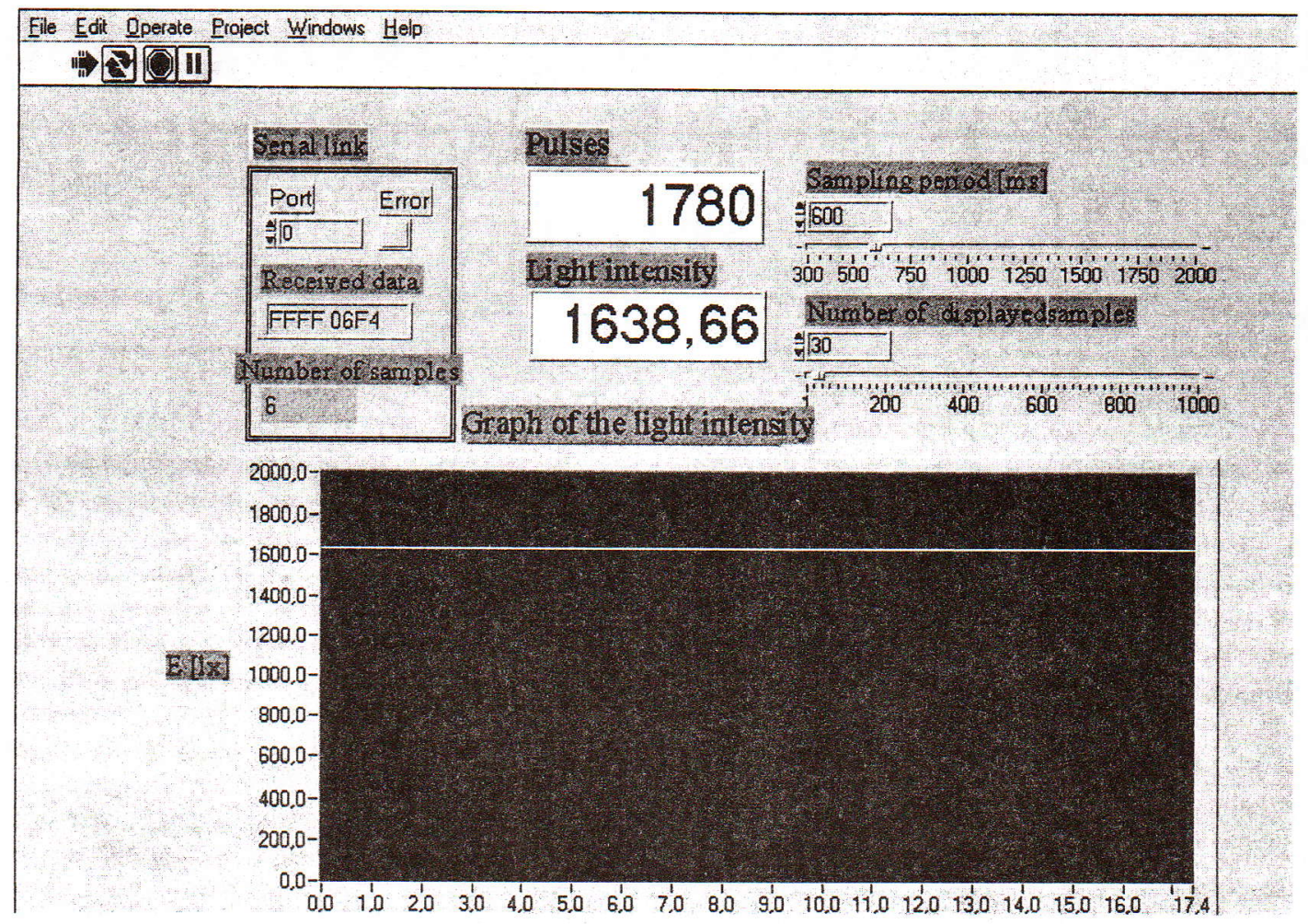

Fig. 6: Window of the processing program in LabView

The experimental relative error $\delta$ as a function of light intensity $E$ is shown in Fig. 5 . In Fig. 5 the maximum of the relative error can be found in the lower part of the range, and its value is $1.5 \%$.

In a practical implementation, conversion of read pulses to light intensity by a polynomial function was realized in LabView. Fig. 6 shows the practical window of the processing program in LabView. The flip-flop sensor was controlled by a voltage pulse according to Fig. 2, while $\delta_{1}, \delta_{2}=18 \mu \mathrm{s}$, $U_{m}=1 \mathrm{~V}$ and $T=40 \mu \mathrm{s}$. The experimental circuit was realized according to Fig. 4 , so that the values of the parameters were set as follows: $R=R_{K}=10 \Omega, R_{1}=R_{2}=6.8 \mathrm{k} \Omega, R_{0}=1.8 \mathrm{k} \Omega$. A 1 PP75 photodiode was used, and the quantization error of the DAC was $10 \mathrm{mV}$.

\section{Conclusions}

A new method for measuring light intensity using a flip-flop has been presented. The method is based on controlling the control pulse by a slow-rise. The validity of the theoretical results was proved by laboratory experiment. In spite of the simple structure of the flip-flop, this system enables light intensity to be measured with a relative error less than $1.5 \%$.

\section{Acknowledgment}

The work presented in this paper was supported by a grant from the Ministry of Education and Academy of Sciences of Slovak Republic (VEGA), under Grant No.1/9030/02.

\section{References}

[1] Lian, W.: Integrated Silicon Flip-flop Sensor. PhD thesis, Technical University of Delft, Netherlands, 1990.

[2] Kollár, M.: Smart Sensors Based on Flip-flop Circuits. PhD thesis, Technical University Košice, 2002 (In Slovak).

[3] Levický, D., Michaeli, L., Špány, V., Pivka, L., Kalakaj, P.: Autocompensative Systems with Flip-flop Sensor. Proceeding of IMEKO $10^{\text {th }}$ Int. Symposium on Development in Digital Measuring Instrumentation, Vol. II., Napoli 1996, p. 665-670.

[4] Michaeli, L.: Modeling of Analog-digital Interfaces. Technical University Košice Press, 2001 (In Slovak).

Martin Kollár

phone: +421556022579

fax: +421556023989

e-mail: Martin.Kollar@tuke.sk

Department of Theoretical Electrotechnics and Electrical Measurement

Technical University of Košice

Park Komenského 3

04389 Košice, Slovak Republic 